\title{
Exosomes derived from miR-92a-3p- overexpressing human mesenchymal stem cells enhance chondrogenesis and suppress cartilage degradation via targeting WNT5A
}

\author{
Guping $\mathrm{MaO}^{\dagger}$, Ziji Zhang $^{\dagger}$, Shu Hu${ }^{\dagger}$, Zhiqi Zhang, Zongkun Chang, Zhiyu Huang, Weiming Liao ${ }^{*}$ and Yan Kang ${ }^{*}$
}

\begin{abstract}
Background: WNT5A is known to be involved in the pathogenesis of osteoarthritis. This study investigated the molecular mechanism of exosomal miR-92a-3p and WNT5A in chondrogenesis and cartilage degeneration.

Methods: Exosomal miR-92a-3p expression was assessed in vitro in a human mesenchymal stem cell (MSC) model of chondrogenesis and in normal and OA primary human chondrocytes (PHCs). MSCs and PHCs were treated with exosomes derived from MSC-miR-92a-3p (MSC-miR-92a-3p-Exos) or its antisense inhibitor (MSC-anti-miR-92a-3pExOs), respectively. Small interfering RNAs (siRNAs) and luciferase reporter assay were used to reveal the molecular role of exosomal miR-92a-3p and WNT5A in chondrogenesis. The protective effect of exosomes in vivo was measured using Safranin-O and Fast Green staining and immunohistochemical staining.

Results: Exosomal miR-92a-3p expression was elevated in the MSC chondrogenic exosome, while it was significantly reduced in the OA chondrocyte-secreted exosome compared with normal cartilage. Treatment with MSC-miR-92a-3p-Exos promoted cartilage proliferation and matrix genes expression in MSCs and PHCs, respectively. In contrast, treatment with MSC-anti-miR-92a-3p-Exos repressed chondrogenic differentiation and reduced cartilage matrix synthesis by enhancing the expression of WNT5A. Luciferase reporter assay demonstrated that miR-92a-3p suppressed the activity of a reporter construct containing the 3'-UTR and inhibited WNT5A expression in both MSCs and PHCs. MSC-miR-92a-3p-Exos inhibit cartilage degradation in the OA mice model.

Conclusions: Our results suggest that exosomal miR-92a-3p regulates cartilage development and homeostasis by directly targeting WNT5A. This indicates that exosomal miR-92a-3p may act as a Wnt inhibitor and exhibits potential as a disease-modifying osteoarthritis drug.
\end{abstract}

Keywords: Exosomes, Human mesenchymal stem cells, WNT5A, miRNA-92a-3p, Osteoarthritis, Chondrocyte

\section{Background}

Osteoarthritis (OA) is strongly associated with joint degenerative diseases, and leads to chronic pain, disability, and economic burden [1]. However, the molecular mechanisms of OA have not yet been fully elucidated. $\mathrm{OA}$ is characterized by the loss of extracellular matrix (ECM) and cartilage destruction. It is widely accepted that the development of $\mathrm{OA}$ is associated with

\footnotetext{
*Correspondence: liaoweiming29@163.com; kangyeah@163.com ${ }^{\dagger}$ Guping Mao, Ziji Zhang and Shu Hu contributed equally to this work. Department of Joint Surgery, First Affiliated Hospital of Sun Yat-sen University, \#58 Zhongshan 2nd Road, Guangzhou 510080, China
}

pro-inflammatory cytokines, which increases the activity of matrix metalloproteinase (MMP) and a disintegrin and metalloproteinase with thrombospondin motifs (ADAMTS) [2, 3].

Recently, increasing evidence has suggested that mesenchymal stem cells (MSCs)-derived exosomes play a vital role in modulating cell migration, proliferation, differentiation, and matrix synthesis [4-6]. In 2010, it was first reported that the exosome was the active agent secreted by MSCs in response to myocardial ischemia reperfusion (I/ R) injury [7]. It has also been reported that MSC exosomes mediate cartilage repair and regeneration by

(c) The Author(s). 2018 Open Access This article is distributed under the terms of the Creative Commons Attribution 4.0 International License (http://creativecommons.org/licenses/by/4.0/), which permits unrestricted use, distribution, and 
enhancing proliferation, attenuating apoptosis, and modulating immune reactivity [8-10]. Exosomes are small, secreted bilipid membrane vesicles of about $50-150 \mathrm{~nm}$, and are thought to act as intercellular communication vehicles. Bioactive lipids, nucleic acids (mRNAs and microRNAs), and proteins are transferred by exosome and play biological responses in cells and recipient cells [7]. Evidence has shown that exosomes have no obvious immunogenicity or tumorigenicity adverse effects [11, 12]. To our knowledge, however, the specific molecular mechanism that causes MSC exosomes to promote cartilage chondrogenesis has not been investigated.

MicroRNAs (miRNAs) are an important component of the exosome and have attracted attention for many years. They bind to the 3 ' - untranslated regions ( 3 ' -UTRs) of target genes and suppress target gene expression [13]. While exosomal miRNAs are widely thought to mediate intercellular communication and gene regulation [14, 15], the specific molecular mechanism of MSC-derived exosomal miRNAs responsible for chondrogenic differentiation and degeneration remain unclear.

WNT5A is considered a non-canonical Wnt protein. Increasing evidence has shown that the development of articular joints, including cartilage, bone, and joint cavities, is highly dependent on Wnt signaling [16-20]. WNT5A has a dual function, acting in both chondrogenic differentiation and cartilage degradation [21]. In the early stage of cartilage formation, it activates the proliferation and inhibits the differentiation of chondrocytes [22-24]. In addition, WNT5A can activate matrix metalloproteinases (MMPs) and reduce cartilage formation and the synthesis of cartilage ECM in the late stage of chondrogenesis and in mature chondrocytes [21, 25]. WNT5A plays a key role in cartilage destruction and degradation in the pathogenesis of OA. Huang et al. showed that WNT5A can promote chondrocyte catabolic activity via non-canonical Wnt signaling in human OA cartilage [25]. Moreover, Ge et al. showed that WNT5A plays an essential role in interleukin (IL)-1 $\beta$-mediated cartilage destruction via the NF- $\mathrm{kB}$ pathway $[26,27]$, and Shi et al. demonstrated that the silencing of WNT5A prevents interleukin (IL)-1 $\beta$-induced collagen type II degradation in rat chondrocytes [28].

In order to elucidate the specific MSC-derived exosomal miRNAs responsible for MSC-mediated chondrogenic differentiation, in the present study, we used a miRNA microarray to determine miRNA expression profiles in exosomes during the chondrogenic differentiation of human MSCs. We observed significant upregulation of the exosomal miR-92a-3p during MSC-induced chondrogenesis. Previous studies we have demonstrated miR-92a-3p plays a key role in chondrogenesis and cartilage degradation via directly targeting noggin3,HDAC2, ADAMTS4, and ADAMTS5 [29-31]. Given the potential role of MSC-derived exosomal miRNAs in regulating cartilage homeostasis and cartilage development, we hypothesized that exosomal miR-92a-3p may play a role in both chondrogenic differentiation and OA pathogenesis. Thus, our study further aimed to determine whether MSC-derived exosomal miR-92a-3p enhances cartilage development and prevents degradation by targeting WNT5A.

\section{Methods}

Human mesenchymal stem cell isolation, culture, and induction of chondrogenesis in micromass culture

Bone marrow samples were obtained from the First Affiliated Hospital of Sun Yat-Sen University. MSCs were isolated as described previously [32]. Bone marrow samples were obtained by iliac crest aspiration from six normal human donors (mean age: 35 years; range: 3238 years; male: 3, female: 3). Human MSCs were cultured in alpha-modified Eagle's medium ( $\alpha$-MEM) (Gibco, Grand Island, NY, USA) supplemented with 10\% fetal bovine serum (FBS; Gibco), $100 \mathrm{IU} / \mathrm{mL}$ penicillin, and $100 \mu \mathrm{g} / \mathrm{mL}$ streptomycin. Cells were cultured at $37^{\circ}$ $\mathrm{C}$ in a $5 \% \mathrm{CO} 2$ atmosphere. Culture media were changed every 3 days. When cultures neared $80 \%$ confluence, cells were detached by treatment with $0.05 \%$ trypsin/ethylenediaminetetraacetic acid (EDTA) and passaged in culture. All hMSCs were used at passage 3 to induce hMSC chondrogenesis by micromass culture, as previously described [16]. Briefly, hMSCs were resuspended at $2 \times 107$ cells $/ \mathrm{mL}$ in incomplete chondrogenic medium [97 mL human mesenchymal stem cell chondrogenic differentiation basal medium, $10 \mu \mathrm{L}$ dexamethasone, $300 \mu \mathrm{L}$ ascorbate, $1 \mathrm{~mL}$ of ITS (insulin, transferrin, selenium) supplement, $100 \mu \mathrm{L}$ sodium pyruvate, and $100 \mu \mathrm{L}$ proline; Cyagen Biosciences, Guangzhou, China]. Droplets of resuspended cells $(12.5 \mu \mathrm{L})$ were carefully transferred to individual wells of a 24-well plate and incubated at $37{ }^{\circ} \mathrm{C}$ for $90 \mathrm{~min}$ to stimulate the adherence of cells to the plate. Droplets were divided into two groups: the first group was cultured in $500 \mu \mathrm{L}$ incomplete chondrogenic medium per well; the second group was cultured in $500 \mu \mathrm{L}$ complete chondrogenic induction medium, prepared by the addition of $10 \mu \mathrm{L}$ transforming growth factor (TGF)- $\beta 3$ to $1 \mathrm{~mL}$ incomplete chondrogenic medium (Cyagen Biosciences). Samples were collected for experiments at selected time points.

\section{Characterization of MSCs and flow cytometry analysis}

To identify the multiple differentiation potential of MSCs, cells were induced to differentiate in osteogenic, adipogenic, or chondrogenic differentiation medium. We used Alizarin Red, Oil Red O, and Alcian Blue staining to identify the three cell types, respectively. Flow cytometry was used to identify the surface antigens of hMSCs. 
CD11b, CD19, CD34, CD45, CD73, CD90, CD105, and HLA-DR (eBioscience, Inc., San Diego, CA, USA) monoclonal antibodies were used for detection. Mouse IgG monoclonal antibody was used as a negative control.

\section{Isolation and identification of exosomes}

Exosome isolation was carried out using ultracentrifugation [33]. In brief, MSC culture supernatants were subjected to successive centrifugations at $3000 \times g(30 \mathrm{~min})$ and $10,000 \times g(30 \mathrm{~min})$. Exosomes were then pelleted at $64,000 \times g$ for $110 \mathrm{~min}$ using an SW28 rotor (Beckman Coulter, Brea, CA, USA). Exosome pellets were resuspended in $0.32 \mathrm{M}$ sucrose and centrifuged at 100,000 $\times g$ for $1 \mathrm{~h}$ (SW60Ti rotor, Beckman Coulter). The exosome pellet was then resuspended in phosphate-buffered saline (PBS). Nanosight 2000 analysis and transmission electron microscopy (TEM) were used to identify exosomes. RNA and proteins were extracted from exosomes using a Total Exosome RNA \& Protein Isolation Kit (Invitrogen, Carlsbad, CA, USA) for further analysis.

Primary chondrocyte collection, isolation, and cell culture Degraded joint cartilage samples were obtained from patients [ $n=6$; mean age: 60.24 years; male: 3 , female: 3$]$ with OA knee joints during total knee replacement operations. Normal cartilage samples were taken from patients $(n=6$; mean age: 54.46 years; male: 3 , female: 3 ) with no previous history of OA or rheumatoid arthritis, who underwent total hip replacement surgery because of femoral neck fractures. The cartilages were dissected away from the subchondral bone and then digested by $4 \mathrm{mg} / \mathrm{mL}$ protease and $0.25 \mathrm{mg} / \mathrm{mL}$ collagenase $\mathrm{P}$ as described previously [30]. Cells were cultured in DMEM/ F-12 (Gibco Life Technologies, Grand Island, NY, USA) containing 5\% fetal bovine serum (FBS; Gibco Life Technologies), $1 \%$ penicillin and streptomycin (Gibco Life Technologies). The chondrocytes were used our in experiments within 3-7 days and without passaging to avoid dedifferentiation.

\section{Chondrocyte migration and proliferation assay}

A scratch wound assay was used to analyze the effect of exosomes secreted by mesenchymal stem cells (MSC-Exos) and exosomes secreted by miR-92a-3p-overexpressing mesenchymal stem cells (MSC-miR-92a-3p-Exos) on the migration of chondrocytes, as described previously [34]. The images were obtained at the same position before and after incubation. Scratched areas were measured using Image-Pro Plus 6.0 software (Media Cybernetics, Bethesda, MD, USA). The effect of MSC-Exos and MSC-miR-92a-3p-Exos on the proliferation of human chondrocytes was evaluated using the Cell Counting Kit-8 (CCK-8; Dojindo, Kyushu Island, Japan) as described previously [34]. Cell proliferation curves were constructed by measuring the amount of formazan dye generated by cellular dehydrogenase activity with a microplate reader at a wave length of $450 \mathrm{~nm}$.

RNA extraction, reverse transcription, and quantitative real-time polymerase chain reaction (qRT-PCR)

RNA extraction and reverse transcription were performed as described previously [31]. Transcript levels were normalized to that of the housekeeping gene glyceraldehyde 3-phosphate dehydrogenase (GAPDH; for mRNA) or the small U6 RNA (for miRNA). The specific primers used for these analyses are listed in Table 1 . Gene expression was calculated using the $2-{ }^{\Delta \Delta} \mathrm{Ct}$ method, and each experiment was performed in triplicate.

\section{Transfection}

The MSCs were transfected with miR-92a-3p mimic or inhibitor (RiboBio, Guangzhou, China) at a concentration of $50 \mathrm{nM}$; they were also transfected with WNT5A siRNA or NC (RiboBio). Lipofectamine 2000 Transfection Reagent (Gibco Life Technologies) was used to transfect cells according to the manufacturer's instructions. Cells were then harvested after $48 \mathrm{~h}$ for quantitative real-time reverse transcription-polymerase chain reaction (qRT-PCR), or after $72 \mathrm{~h}$ for western blot analysis.

\section{Western blot analysis}

Western blot analysis was carried out as described previously [31]. Membranes were incubated with primary antibodies against WNT5A and RUNX2 (1:1000 dilution, Cell Signaling Technology); GAPDH (1:3000, Cell Signaling Technology); aggrecan, COL2A1, and MMP13 (1:1000, Abcam, Cambridge, MA, USA); and SOX9 (1:2000, EMD Millipore, Burlington, MA, USA). The blots were then incubated with appropriate secondary antibodies (1:3000 dilution, Cell Signaling Technology) at $4{ }^{\circ} \mathrm{C}$ overnight, after which they were developed with an ECL chemiluminescence Kit (EMD Millipore).

Immunohistochemical analysis, histology staining, and in situ hybridization

Immunohistochemical analysis was performed as described previously [31]. Briefly, samples were fixed in $4 \%$ paraformaldehyde (Sigma-Aldrich, St. Louis, MO, USA), decalcified, embedded in paraffin, and cut into $5-\mu \mathrm{m}$ sections that were deparaffinized, rehydrated, and then stained with Safranin O/Fast Green. COL2A1, aggrecan, MMP13, and WNT5A expression was analyzed by immunohistochemistry. For in situ hybridization of miR-92a-3p expression, tissues were subsequently dehydrated with a graded series of ethanol, embedded in paraffin, and cut into 5 - $\mu$ m-thick sections. Sections were subjected to in situ hybridization analysis using 
Table 1 Primers for quantitative real-time polymerase chain reaction (qRT-PCR)

\begin{tabular}{|c|c|c|}
\hline Gene & & Primer sequence $\left(5^{\prime}-3^{\prime}\right)$ \\
\hline hsa-COL2A1 & $\mathrm{F}$ & GCACCTGCAGAGACCTGAAAC \\
\hline hsa-COL2A1 & $\mathrm{R}$ & GCAAGTCTCGCCAGTCTCCA \\
\hline hsa-COL9A1 & $\mathrm{F}$ & GGCAGTAGAGGAGAATTAGGACC \\
\hline hsa-COL9A1 & $\mathrm{R}$ & GTTCACCGACTACACCCCTG \\
\hline hsa-COL10A1 & $\mathrm{F}$ & CATAAAAGGCCCACTACCCAAC \\
\hline hsa-COL10A1 & $\mathrm{R}$ & АCCTTGCTCTCCTCTTACTGC \\
\hline hsa-SOX9 & $\mathrm{F}$ & GGAGATGAAATCTGTTCTGGGAATG \\
\hline hsa-SOX9 & $\mathrm{R}$ & TTGAAGGTTAACTGCTGGTGTTCTG \\
\hline hsa-RUNX2 & $\mathrm{F}$ & CACTGGCGCTGCAACAAGA \\
\hline hsa-RUNX2 & $\mathrm{R}$ & CATTCCGGAGCTCAGCAGAATAA \\
\hline hsa-WNT5A & $\mathrm{F}$ & ATTCTTGGTGGTCGCTAGGTA \\
\hline hsa-WNT5A & $\mathrm{R}$ & CGCCTTCTCCGATGTACTGC \\
\hline hsa-COMP & $\mathrm{F}$ & GATCACGTTCCTGAAAAACACG \\
\hline hsa-COMP & $\mathrm{R}$ & GCTCTCCGTCTGGATGCAG \\
\hline hsa-Aggrecan & $\mathrm{F}$ & GATGTTCCCTGCAATTACCACCTC \\
\hline hsa-Aggrecan & $\mathrm{R}$ & TGATCTCATACCGGTCCTTCTTCTG \\
\hline hsa-MMP-13 & $\mathrm{F}$ & TCCTGATGTGGGTGAATACAATG \\
\hline hsa-MMP-13 & $\mathrm{R}$ & GCCATCGTGAAGTCTGGTAAAAT \\
\hline hsa-GAPDH & $\mathrm{F}$ & GCACCGTCAAGGCTGAGAAC \\
\hline hsa-GAPDH & R & TGGTGAAGACGCCAGTGGA \\
\hline hsa/mmu-U6 & $\mathrm{F}$ & CTCGCTTCGGCAGCACA \\
\hline hsa/mmu-U6 & R & AACGCTTCACGAATTTGCGT \\
\hline hsa-miR-92a-3p & $\mathrm{F}$ & CACTTGTCCCGGCCTGTAAA \\
\hline Mmu-miR-92a-3p & F & TATTGCACTTGTCCCGGCCTG \\
\hline Mmu-WNT5A & $\mathrm{F}$ & ATGCAGTACATTGGAGAAGGTG \\
\hline Mmu-WNT5A & $\mathrm{R}$ & CGTCTCTCGGCTGCCTATTT \\
\hline Mmu-MMP13 & $\mathrm{F}$ & ATGCATTCAGCTATCCTGGCCA \\
\hline Mmu-MMP13 & $\mathrm{R}$ & AAGATTGCATTTCTCGGAGCCTG \\
\hline Mmu-COL2A1 & $\mathrm{F}$ & CCCGCCTTCCCATTATTGAC \\
\hline Mmu-COL2A1 & $\mathrm{R}$ & GGGAGGACGGTTGGGTATCA \\
\hline Mmu-Aggrecan & $\mathrm{F}$ & ATTCCACACGCTACACCCTG \\
\hline Mmu-Aggrecan & $\mathrm{R}$ & TGGATGGGGTATCTGACTGTC \\
\hline Mmu-GAPDH & $\mathrm{F}$ & TGTGTCCGTCGTGGATCTGA \\
\hline Mmu-GAPDH & $\mathrm{R}$ & TTGCTGTTGAAGTCGCAGGAG \\
\hline
\end{tabular}

miR-92a-3p-specific probe (Exiqon, Copenhagen, Denmark), as described in our previous study [31].

\section{Luciferase constructs and reporter assay}

The DNA sequence of the WNT5A 3'-UTR was amplified by PCR using the following primers: forward, 5' -ATAGGCCGGCATAGACGCGTTAGGCAGGTTGG CTTTCATATC-3', and reverse, 5' -AAAGATCCTTTAT TAAGCTTGTGTGAACAGGGAAATTAGATC-3'. The seed sequences were mutated using standard PCR techniques with the following primers: forward, 5'-GTATTCCGATGTAAAAACACAATGAACCTTTA GTTTC-3', and reverse, 5'-GTGTTTTTACATCGGAA TACAAGTTATTGTGCTTTTCAAA-3'. The amplified DNA sequences were inserted into the pmiR-RB-REPORT Vector (OBIO, Shanghai, China) to generate wild-type or mutant WNT5A 3'-UTR luciferase vectors. For the dual luciferase assay, $1.2 \times 10^{4}$ HEK293 cells in a 96-well plate were transfected with $50 \mathrm{nM}$ miR-92a-3p or miR-NC (RiboBio). The cells were then co-transfected with $2 \mu \mathrm{g} / \mathrm{mL}$ of the wild-type or mutant WNT5A 3'-UTR vector. Luciferase activity was measured $48 \mathrm{~h}$ post-transfection using the Dual-Luciferase Reporter Assay System (Promega, Madison, WI, USA). Firefly luciferase activity was then normalized to the corresponding Renilla luciferase activity. Luciferase assays were performed in quadruplicate and repeated in three independent experiments.

\section{Collagenase-induced OA model}

All procedures were approved by the Animal Research Committee of the First Affiliated Hospital of Sun Yat-sen University. Six-week-old female C57B/L10 mice were randomized into four groups: normal group $(n=$ 10), MSC-Exos group $(\mathrm{n}=10)$, MSC-miR-92a-3p-Exos group $(n=10)$, and OA group $(n=10)$. The following operations must be performed in a sterile environment to avoid joint infection. On day 0 , the mice of the MSC-Exos, MSC-miR-92a-3p-Exos, and OA groups were induced with the OA model with collagenase VII (12 U of collagenase VII in $8 \mu \mathrm{l}$ saline, Clostridium histolyticum; Sigma-Aldrich) as described previously [34]. Mice in the normal groups were injected with $8 \mu \mathrm{l}$ saline as control. On days 7, 14, and 21, the MSC-Exos and MSC-miR-92a-3p-Exos groups were injected with $15 \mu \mathrm{l}$ MSC-Exos $(500 \mu \mathrm{g} / \mathrm{mL})$ or $15 \mu \mathrm{l} \mathrm{MSC-miR-92a-3p-Exos}$ in PBS $(500 \mu \mathrm{g} / \mathrm{mL})$. Mice in the OA and normal groups were injected with $15 \mu \mathrm{l}$ PBS at each time point. On day 28 , mice were euthanatized for further analysis.

\section{Statistical analysis}

All experiments were performed with at least three biological replicates. Data were expressed as the mean \pm standard deviations (SD). Both parametric and nonparametric inferential statistics were utilized in this study depending on whether the data was normally distributed. The independent $t$ test and Mann-Whitney $U$ test were used to identify differences between groups as appropriate. One-way analysis of variance (ANOVA) and Kruskal-Wallis tests were carried out for multiple group comparisons. Data analyses were performed using SPSS Version 20 (IBM Corporation, Armonk, NY, USA). Statistical significance was determined at level of $P<0.05$. 


\section{Results}

Identification of MSCs and MSC-Exos

MSCs were extracted during iliac crest aspiration and were isolated and identified at passage 5 (P5) for use in subsequent experiments. After reaching $80-90 \%$ confluence, MSCs showed a robust proliferation capability and appeared to be a relatively homogeneous population of spindle-shaped cells (Fig. 1a). The MSCs could readily be induced to differentiate into the osteogenic, adiopogenic, and chondrogenic lineages, when cultured in their respective medium. Osteogenic potential was confirmed by the staining of calcium mineral deposits with Alizarin Red (Fig. 1b, left panel), while adipogenic potential was evaluated by the observation of small cytoplasmic lipid droplets stained with Oil Red O (Fig. 1b, middle panel). Chondrogenic potential was confirmed by sectioning beads and staining sulfated glycosaminoglycans using Alcian Blue (Fig. 1b, right panel). Following the criteria for identifying stem cells, we analyzed the surface markers of the MSCs. Analysis of surface antigen expression using flow cytometry demonstrated that the MSCs were positive for CD73, CD90, and CD105 and negative for CD11b, CD19, CD34, CD45, and HLA-DR (Fig. 1c). Nanosight analysis showed that the size of the majority of MSC-Exos was approximately 50-150 nm (Fig. 1d, e). TEM clearly revealed that MSC-Exos exhibited a cup-shaped or round morphology with a diameter of 50-150 nm (Fig. 1f). Western blotting analyses indicated that the MSC-Exos expressed exosomal markers such as CD9, CD63, CD81, and HSP70 (Fig. 1g).

\section{MiRNA expression profiles in exosomes before and after chondrogenic differentiation of MSCs}

The expression profiles of miRNAs in exosomes before and after chondrogenic differentiation were detected using a miRNA microarray. SAM statistical software was used to identify differentially expressed miRNAs of exosomes between undifferentiated MSCs and chondrogenically differentiated MSCs. The differentially expressed miRNAs from all three paired samples are shown in
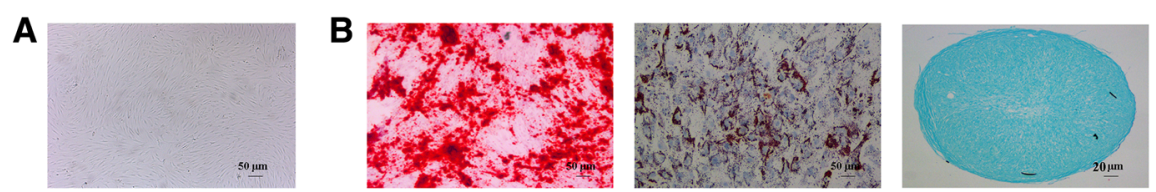

C
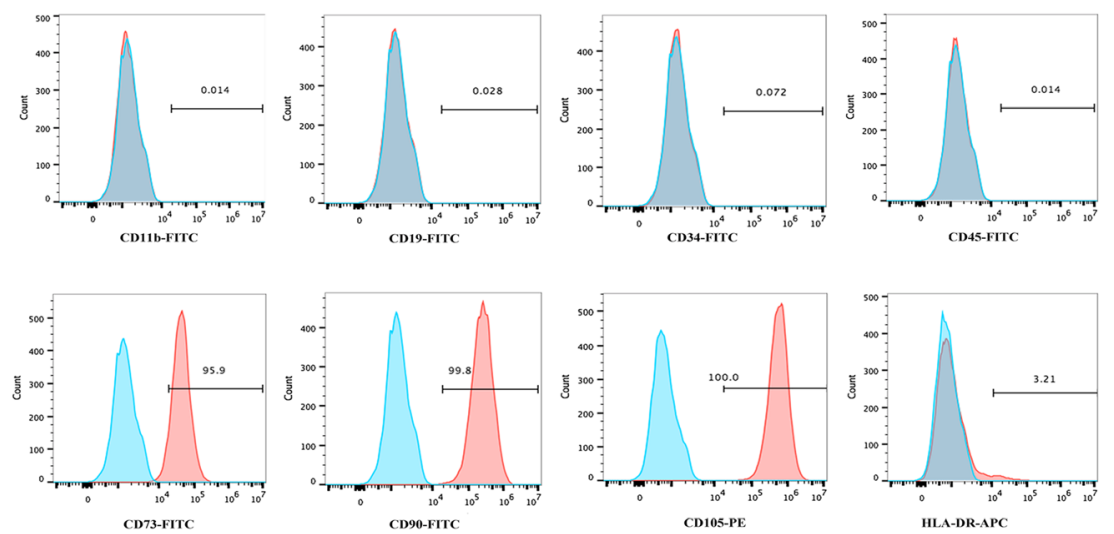

D

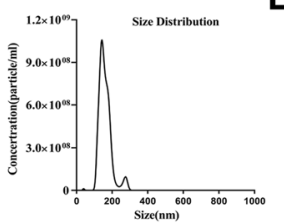

E

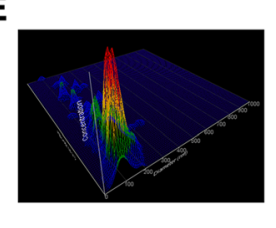

$\mathbf{F}$

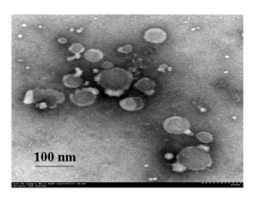

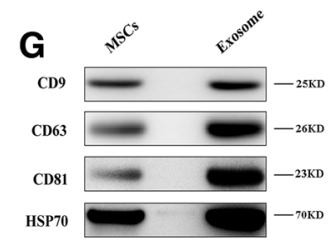

Fig. 1 Characterization of human mesenchymal stem cells (MSCs) and exosomes. a MSCs exhibited a representative spindle-like morphology. $\mathbf{b}$ MSCs exhibited multidifferentiation capacity for osteogenesis, adipogenesis, and chondrogenesis. c Flow cytometric analysis of mesenchymal positive markers, such as CD73, CD90, and CD105, and negative markers, such as CD11b, CD19, CD34, CD45, and HLA-DR. Blue histograms represent the isotype controls, and the red solid peak represents the marker indicated. This experiment was repeated three times independently. d-e Particle size distribution of exosomes was measured by Nanosight. $\mathbf{f}$ Morphology of exosomes observed by transmission electron microscopy (TEM). $\mathbf{g}$ Exosome surface markers (CD9, CD63, CD81, and HSP70) measured using western blotting. This experiment was repeated three times independently, and representative results are shown 
Fig. 2a-b and Additional file 1. Among the miRNAs that were consistently differentially expressed in all three paired samples, 36 were upregulated (fold change >2) and 105 were downregulated (fold change $<-2$ ) in chondro-differentiated MSC-Exos compared to levels in MSC-Exos, as shown in Additional file 1. Among the top ten most significantly differentially expressed exosomal miRNAs (Fig. 2c), exosomal miR-92a-3p was upregulated 7.89-fold in exosomes after chondrogenic differentiation of MSCs. These data indicate that exosomal miRNAs play an important role in cartilage development.

\section{Chondrocyte proliferation and migration assays}

Proliferation was evaluated following the stimulation of chondrocytes with various doses of MSC-Exos, MSC-miR -92a-3p-Exos, or vehicle (PBS) as a control. At a concentration of $200 \mu \mathrm{g}$ exosomes/mL, chondrocytes cultured with either MSC-Exos or MSC-miR-92a-3p-Exos showed greater proliferation than those incubated with PBS or other doses of exosomes. Moreover, the MSC-miR-92a-3p-Exos had a more potent effect on chondrocyte proliferation than the MSC-Exos (Fig. 2d). The chondrocytes were treated with $200 \mu \mathrm{g}$ exosomes/mL MSC-Exos or MSC-miR-92a-3p-Exos. Scratch wound assays indicated that while both MSC-Exos and MSC-miR-92a-3p-Exos significantly enhanced the motility of chondrocytes, MSC-miR-92a-3p-Exos were more effective than MSC-Exos in increasing motility at 24 and $48 \mathrm{~h}$ (Fig. 2e, f).

Opposing expression patterns of exosomal miR-92a-3p and WNT5A during chondrogenic differentiation of MSCs The MSCs were induced to differentiate into chondrocytes in vitro using TGF- $\beta 3$. We observed a rapid upregulation of exosomal miR-92a-3p in chondrogenic MSCs beginning on day 7 , which peaked at 21 days and was followed by a marked decrease in expression at day 28 (Fig. 3a. A). Concurrently, there was a significant
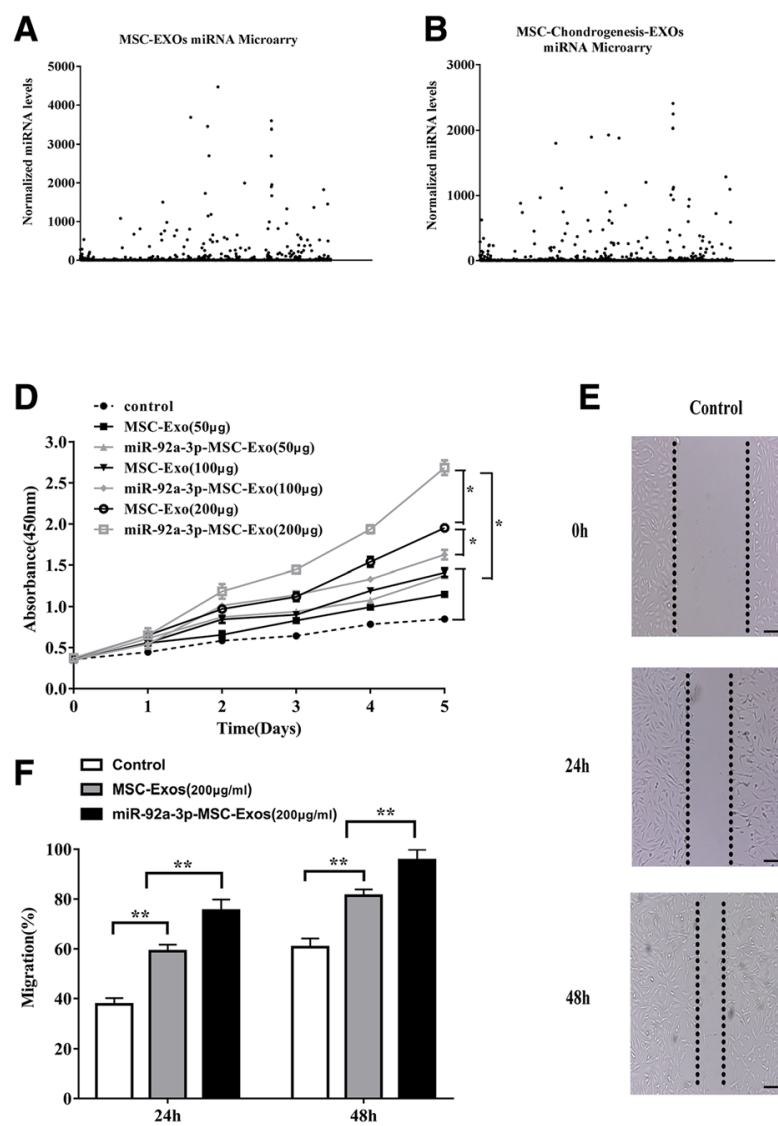

$\mathbf{E}$

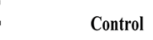

$0 \mathrm{~h}$
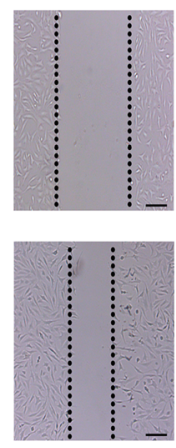

$48 \mathrm{~h}$

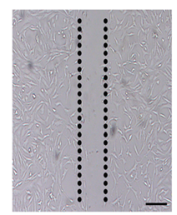

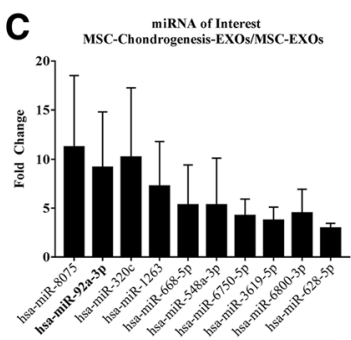
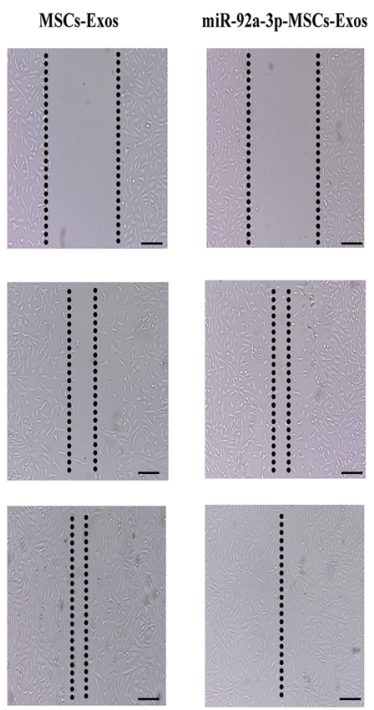

Fig. 2 Effects of miR-92a-3p-MSC-Exos on migration and proliferation in chondrocytes. a-b Normalized gene expression levels in MSC-Exos and chondro-differentiated MSC-Exos detected by miRNA microarray. Three independent samples were used. c Gene expression levels of highly expressed genes in MSC-chondro-differentiated-Exo vs MSC-Exos and gene expression levels of miR-92a-3p. d MSC-Exos and miR-92a-3p-MSCExos were treated with chondrocytes at different doses. Quantitative data are presented as means \pm standard deviations of three independent experiments. ${ }^{*} P<0.05$. e Light microscopy images of scratch wound assays. $\mathbf{f}$ Quantitative analysis of migration rates at 24 and $48 \mathrm{~h}$. Quantitative data are presented as means \pm standard deviations of three independent experiments. ${ }^{*} P<0.01$ 


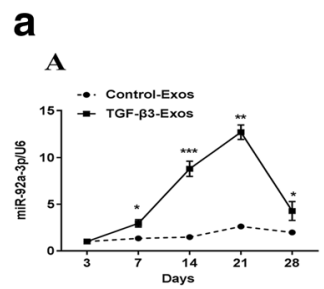

b
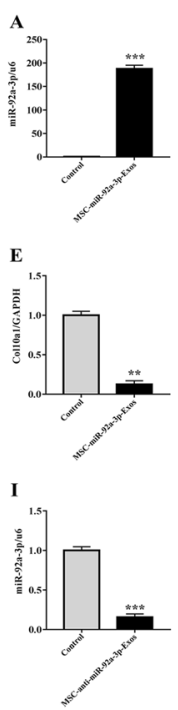

M

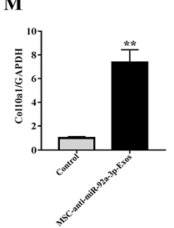

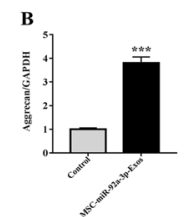
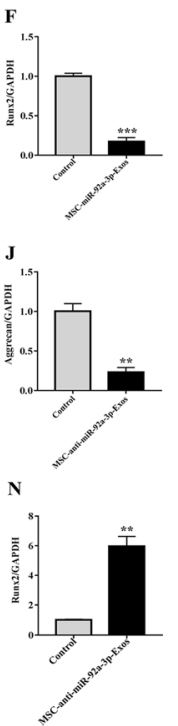
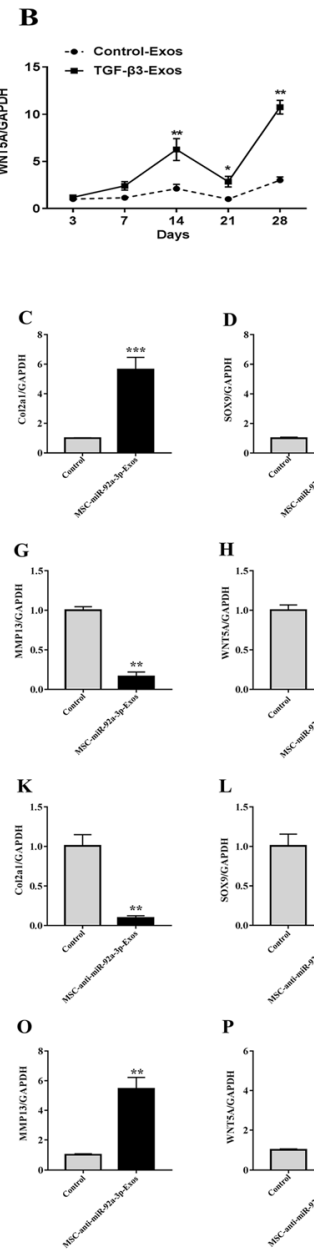

C
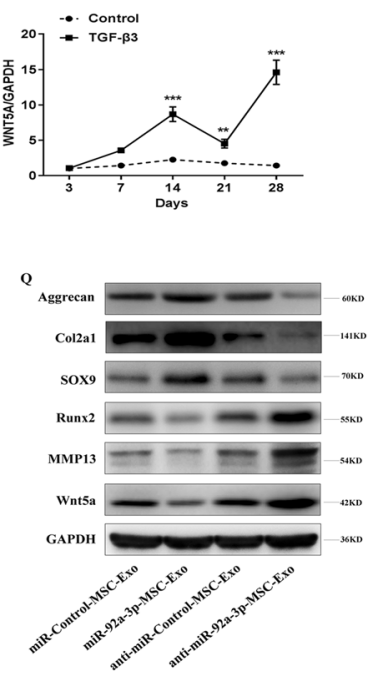

Fig. 3 a Expression of miR-92a-3p and WNT5A mRNA in MSC -chondro-differentiated-Exos. MSCs were induced to undergo chondrogenesis with TGF- 33 for 3, 7, 14, 21, and 28 days as indicated (solid lines). Gene expression levels of miR-92a-3p (A) and WNT5A (B) in chondro-differentiated MSC-Exos and WNT5A (C) in chondro-differentiated MSCs were determined by qRT-PCR. MSCs cultured without TGF- $\beta 3$ at corresponding time points served as negative controls (broken lines). Quantitative data are presented as means \pm standard deviations of three independent experiments. U6 and GAPDH were used as endogenous controls. Data are presented as means \pm standard deviations of six samples. ${ }^{*} P<0.05$, ${ }^{*} P<0.01,{ }^{* *} P<0.001$. b MSC-miR-92a-3p-Exos regulate cartilage-specific gene expression during chondrogenesis. MSCs were treated with TGF- $\beta 3$ to induce chondrogenesis and treated with MSC-miR-92a-3p-Exos or MSC-anti-miR-92a-3p-Exos. Expression levels of miR-92a-3p (A, I) were determined by qRT-PCR. Expression levels of aggrecan (B, J, Q), COL2A1 (C, K, Q), SOX9 (D, L, Q), COL10A1 (E, M), RUNX2 (F, N, Q), MMP13 $(G, O, Q)$, and WNT5A (H, P, Q) were determined by both qRT-PCR and western blotting. U6 and GAPDH were used as endogenous controls. Data represented as means \pm standard deviations of six samples. ${ }^{* *} P<0.01,{ }^{* *} P<0.001$

upregulation of WNT5A in exosomes and tissues on days 21-28, suggesting that miR-92a-3p may affect WNT5A expression (Fig. 3a. B, C).

\section{Exosomal miR-92a-3p regulates cartilage development in MSCs during chondrogenesis}

To further investigate whether exosomal miR-92a-3p regulates chondrogenesis of MSCs, we overexpressed or inhibited miR-92a-3p in MSCs. The MSCs were transfected with MSC-miR-92a-3p-Exos or MSC-anti-miR-92a-3p-Exos. Next, MSC cultures near $90 \%$ confluence were induced to differentiate into chondrocytes and were incubated with
MSC-miR-92a-3p-Exos or MSC-anti-miR-92a-3p-Exos in micromass for 14 days (Fig. 3b). The mRNA and protein expression levels of aggrecan, COL2A1, and SOX9 (Fig. 3b.B-D) were significantly upregulated and those of COL10A1, RUNX2, MMP13, and WNT5A were significantly downregulated in the MSC-miR-92a-3p-Exos treatment as assessed by qRT-PCR and western blotting (Fig. 3b.Q). In contrast, COL10A1, RUNX2, MMP13, and WNT5A levels were significantly upregulated (Fig. 3b.N-P and Fig. 3b.Q) and COL2A1, aggrecan, and SOX9 were significantly downregulated (Fig. 3b.J-L and Fig. 3b.Q) in MSC-anti-miR-92a-3p-Exos. These data indicate that 
miR-92a-3p may target WNT5A to promote SOX9 and aggrecan expression and enhance cartilage development.

\section{Expression levels of exosomal miR-92a-3p and WNT5A in normal and $O A$ cartilage}

To determine the expression of exosomal miR-92a-3p during the progression of $\mathrm{OA}$, we compared its expression levels in normal and OA cartilage-secreted exosomes. Exosomal miR-92a-3p expression was significantly reduced in OA cartilage compared with levels in normal cartilage (Fig. 4a). However, OA cartilage exhibited higher levels of WNT5A mRNA (Fig. 4b, c) and protein compared with levels in normal cartilage (Fig. 4d, e).

\section{Exosomal miR-92a-3p maintains the function of articular chondrocytes}

To determine the effect of exosomal miR-92a-3p on chondrocyte function, we treated chondrocytes with MSC-miR-92a-3p-Exos or MSC-anti-miR-92a-3p-Exos $(200 \mu \mathrm{g}$ exosomes $/ \mathrm{mL})$. We found that MSC-miR-92a-3p -Exos significantly upregulated the mRNA and protein expression levels of aggrecan, COL2A1, COL9A1, COMP, and SOX9 and decreased the expression levels of COL10A1, RUNX2, MMP13, and WNT5A (Fig. 5). In contrast, MSC-anti-miR-92a-3p-Exos accelerated cartilage matrix degradation and increased WNT5A expression. These data indicate that MSC-miR-92a-3p-Exos may slow the progression of $\mathrm{OA}$ and maintain cartilage stability.
SiWNT5A promotes cartilage-specific gene expression To perform the converse of the above experiments, a siRNA approach was used to reduce the levels of WNT5A. Chondrocytes were transfected with siWNT5A, which resulted in the downregulation of WNT5A mRNA levels, as shown by qRT-PCR (Fig. 6a). This reduction was well correlated with a decrease in cartilage WNT5A protein expression, as well as increases in the expression of COL2A1, aggrecan, and SOX9, as shown in Fig. 6b. These data indicate that the downregulation of WNT5A promotes increases in the expression of cartilage-specific proteins.

\section{MiR-92a-3p directly targets the 3'-UTR of WNT5A mRNA}

To further clarify the molecular mechanisms that underlie the regulation of WNT5A expression by miR-92a-3p, we analyzed the sequence of the 3 '-UTR of the human WNT5A gene. Bioinformatics software such as Targetscan (http://www.targetscan.org) and miRanda (http:// www.microrna.org) revealed that the 3 '-UTR of human WNT5A contains a potential miR-92a-3p binding site (Fig. 6c). Luciferase reporter assays with the wild-type or mutant 3'-UTR of WNT5A were performed in the presence or absence of miR-92a-3p overexpression. Transfection with miR-92a-3p resulted in reduced luciferase activity (indicating reduced transcription of WNT5A) by binding to the wild-type $3^{\prime}$-UTR, while the mutant $3^{\prime}$-UTR sequence prevented the binding of miR-92a-3p.
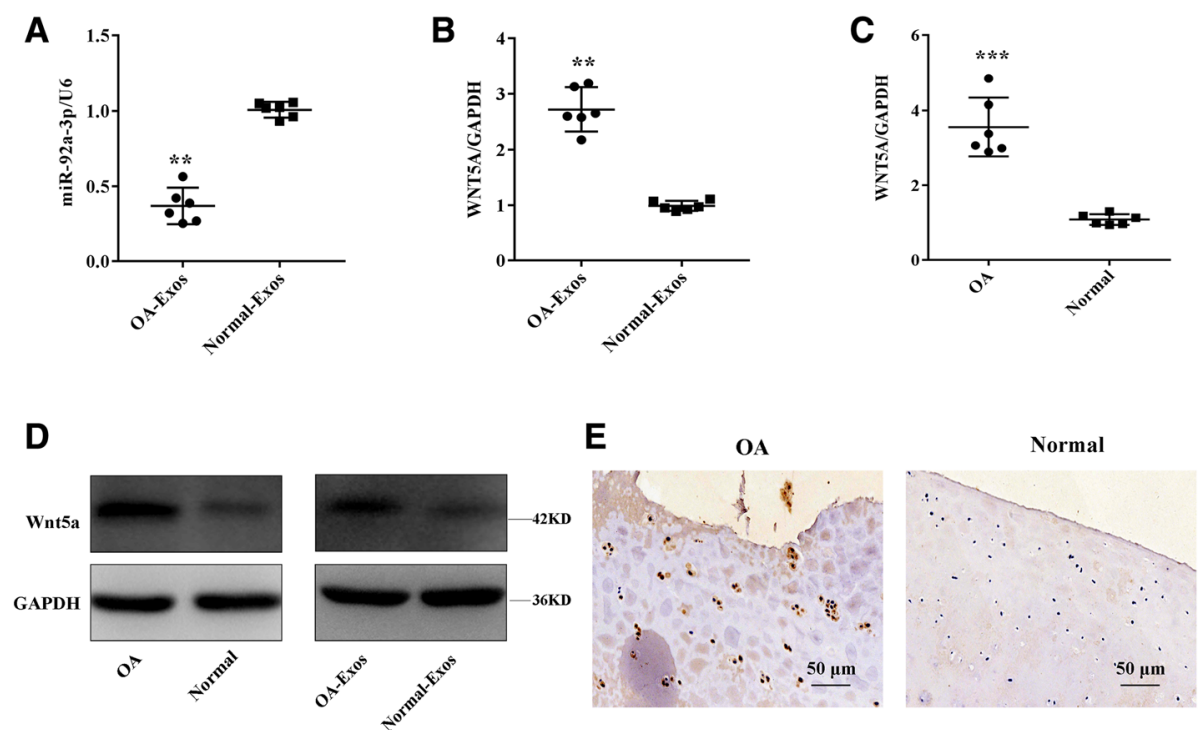

Fig. 4 Expression of miR-92a-3p and WNT5A in normal and OA cartilage. Relative miR-92a-3p and WNT5A (a, b) mRNA levels in normal and OA cartilage-secreted exosomes and (c) WNT5A mRNA levels in normal and OA cartilage tissues were determined by qRT-PCR. U6 and GAPDH were used as endogenous controls. Each dot represents a value from a single experiment involving a single donor. The bar shows the mean and $95 \%$ confidence intervals of the values from six different donors per group. ${ }^{* *} P<0.01,{ }^{* * *} P<0.001$. WNT5A protein levels in normal cartilage and OA cartilage-secreted exosomes or tissues were determined by western blotting and immunohistochemistry using anti-WNT5A monoclonal antibody (d, e). GAPDH was used as an endogenous control. Data shown are representative of results of six normal and OA cartilage samples. Scale bar $50 \mu \mathrm{m}$ 

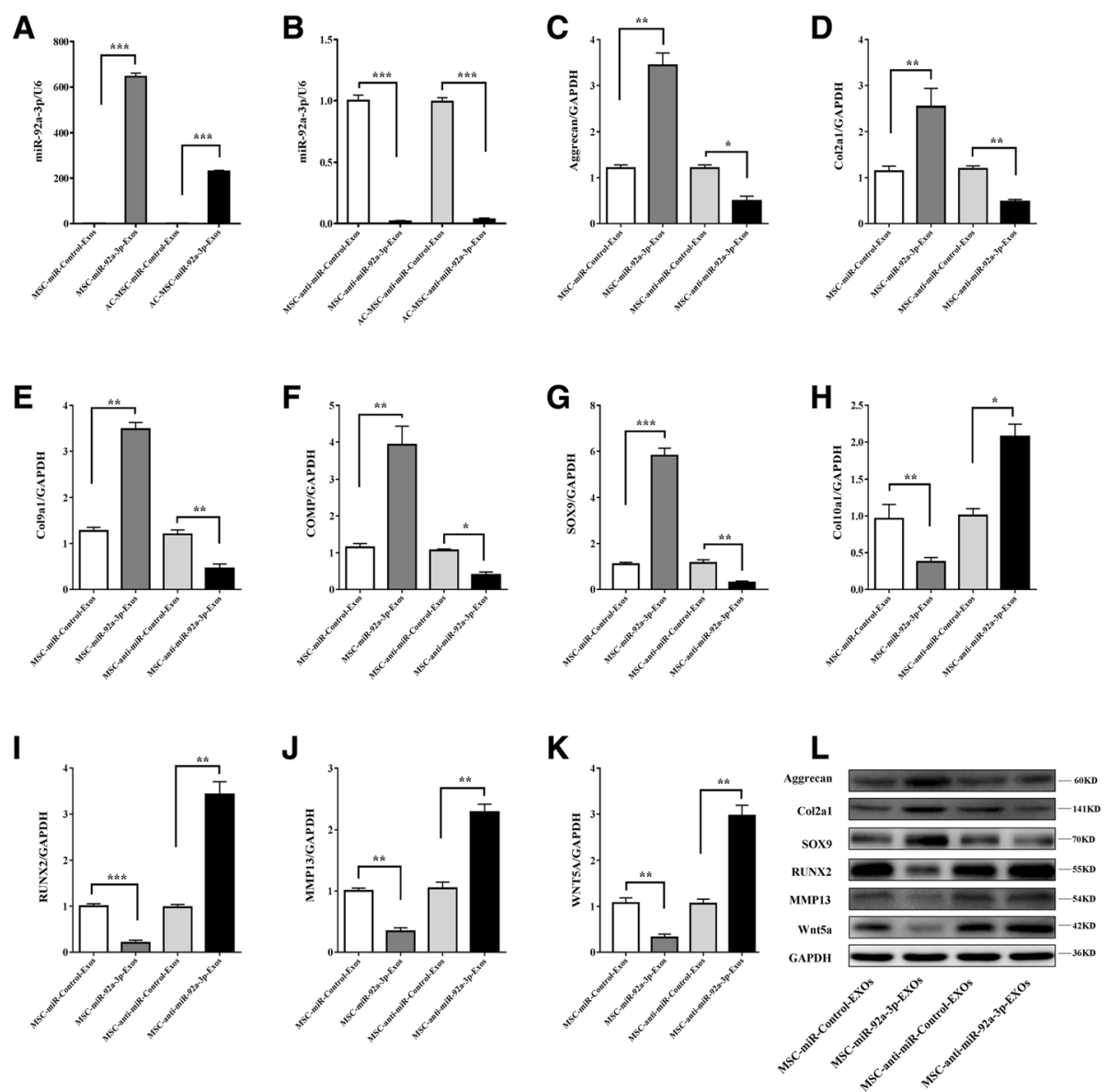

Fig. 5 Responses of articular chondrocytes to stimulation by MSC-miR-92a-3p-Exos. MiR-92a-3p (a, b) expression level changes in exosomes after treatment of chondrocytes with MSC-miR-Control-Exos, MSC-miR-92a-3p-Exos, MSC-anti-miR-Control-Exos or MSC-anti-miR-92a-3p-Exos were determined by qRT-PCR. U6 was used as an endogenous control. Gene expression levels of aggrecan (c), COL2A1 (d), COL9A1 (e), COMP (f), SOX9 (g), COL10A1 (h), RUNX2(i), MMP13(j), and WNT5A(k) were estimated by qRT-PCR. Quantitative data are presented as means \pm standard deviations of three independent experiments. GAPDH was used as an internal control. ${ }^{*} P<0.05,{ }^{*} P<0.01,{ }^{* * *} P<0.001$. I Protein expression levels of aggrecan, col2a1, SOX9, RUNX2, MMP13, and Wnt5a were visualized by western blotting. GAPDH was used as an endogenous control

This suggests that WNT5A is a target of miR-92a3p-mediated repression (Fig. 6d, e).

\section{MSC-miR-92a-3p-Exos inhibits cartilage degradation}

To investigate the role of exosomal miR-92a-3p in osteoarthritis in vivo, we built the OA mice model (Fig. 7). In the OA group, the cartilage matrix consisting of col2a1 and aggrecan in cartilage were obviously decreased, while expression of wnt5a and MMP13 were upregulated on the cartilage surface. In the OA + MSC-Exos group, cartilage matrix loss was much less severe compared to the OA group. In the OA + MSC-miR-92a-3p-Exos group, cartilage matrix was still present but the severity was very mild. The col2a1 and aggrecan were slightly less than in the normal groups but significantly better than in the OA group or the OA + MSC-Exos group. These histological staining results are consistent with WNT5A, MMP13,
COL2A1, and aggrecan mRNA and protein expression. These data indicated that MSC-miR-92a-3p-Exos inhibit the progression of early $\mathrm{OA}$ and prevented the severe damage to knee articular cartilage in the OA mice model.

\section{Discussion}

Osteoarthritis (OA) is a common, debilitating disease that is associated with a large social and economic burden. Disease progression is usually slow but can ultimately lead to joint failure with pain and disability [35, 36]. Current therapies only relieve the symptoms, but cannot stop or reverse cartilage degeneration and destruction [37, 38]. Previous studies showed that MSC exosomes, which contain miRNAs, mRNAs, and proteins, are likely to be important mediators in cell-cell communication to induce changes in cell functions and processes [39, 40]. Exosomal miRNAs are important 
A

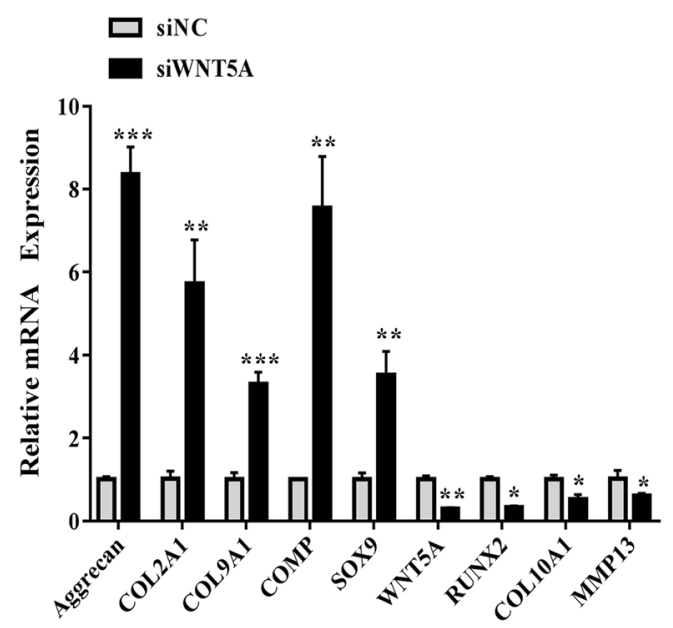

B

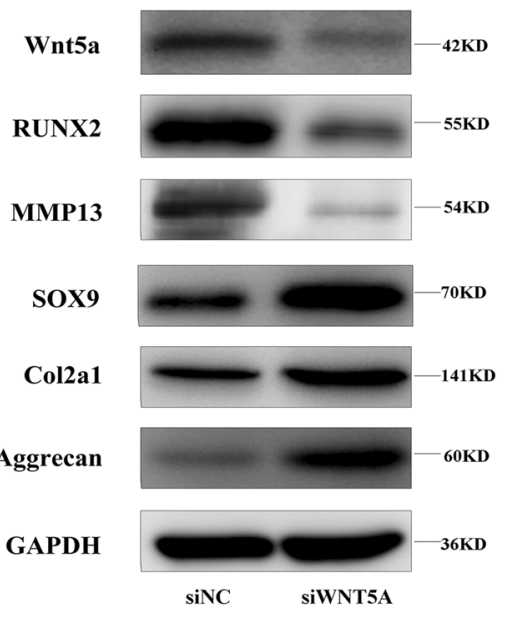

C Position 3058-3064 of WNT5A 3'UTR hsa-miR-92a-3p

\section{D}

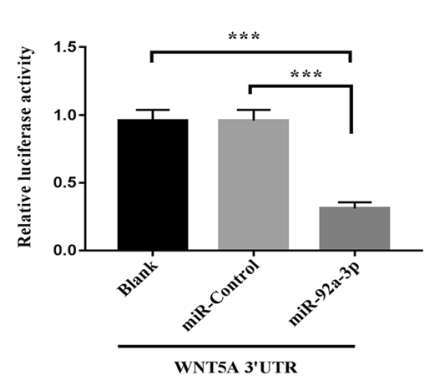

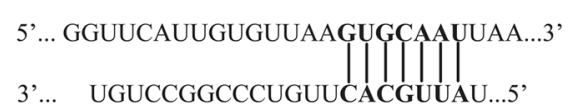

E

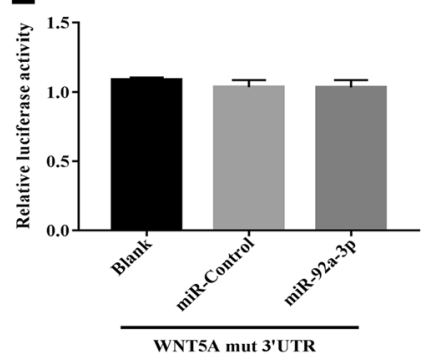

Fig. 6 PHCs were transfected with siWNT5A. a The gene expression levels of aggrecan, COL2A1, COMP, COL9A1, SOX9, RUNX2, COL10A1, MMP13, and WNT5A were determined by qRT-PCR. Quantitative data are presented as means \pm standard deviations of three independent experiments. GAPDH was used as an internal control. ${ }^{*} P<0.05$, ${ }^{* *} P<0.01$, ${ }^{* *} P<0.001$. b Expression of WNT5A, RUNX2, MMP13, SOX9, COL2A1, and aggrecan as visualized by western blot. GAPDH was used as an internal control. c Alignment of miR-92a-3p and the 3'-UTR of WNT5A, a potential miR-92a-3p target. $\mathbf{d}$, e A luciferase reporter carrying the 3'-UTR of wild-type (Luc-WNT5A-UTR) or mutant (Luc-WNT5A-UTR-mut) WNT5A was introduced into 293 T cells along with negative miR-control (NC) or miR-92a-3p. Cells were harvested $48 \mathrm{~h}$ later for luciferase assays. Quantitative data are presented as means \pm standard deviations of three independent experiments. ${ }^{* *} P<0.001$

components of MSC exosomes, and modulate a variety of injuries and diseases, including OA. For example, some of the miRNAs found in MSC exosomes, such as miR-23b and miR-320may be therapeutic for their roles in modulating matrix synthesis [41, 42].

In this study, we verified the presence of miRNAs in exosomes isolated from the supernatant of MSC culture. We found that miR-8075, miR-92a-3p, miR-320c, miR-1263, miR-668-5p, miR-548a-3p, miR-6750-5p, miR-3619-5p, and miR-628-5p were significantly upregulated in three paired exosomal samples from MSCs induced to undergo chondrogenesis. In contrast, miR-1225-5p, miR-7150, miR-466, miR-7107-5p, and miR-329-3p were significantly downregulated in these exosomes. Furthermore, we are the first to discover that exosomal miR-92a-3p mediates cartilage development and degradation via WNT5A. Our data showed that exosomal miR-92a-3p was expressed in vitro, particularly during the late stage of chondrogenic differentiation. Previous studies have shown that miR-92a-3p enhances SOX9 and COL2A1 expression, directly targets $H D A C 2$, and delays the progression of $\mathrm{OA}$ by suppressing ADAMTS4 and ADAMTS5 [30, 31].

In further experiments, we found that treatment with MSC-miR-92a-3p-Exos promoted cartilage development when MSCs were induced to differentiate into chondrocytes. Furthermore, WNT5A was validated as a target of miR-92a-3p through bioinformatics analysis, western blot assays, and luciferase reporter assays. Moreover, treatment with MSC-anti-miR-92a-3p-Exos enhanced WNT5A expression in PHCs and was associated with 


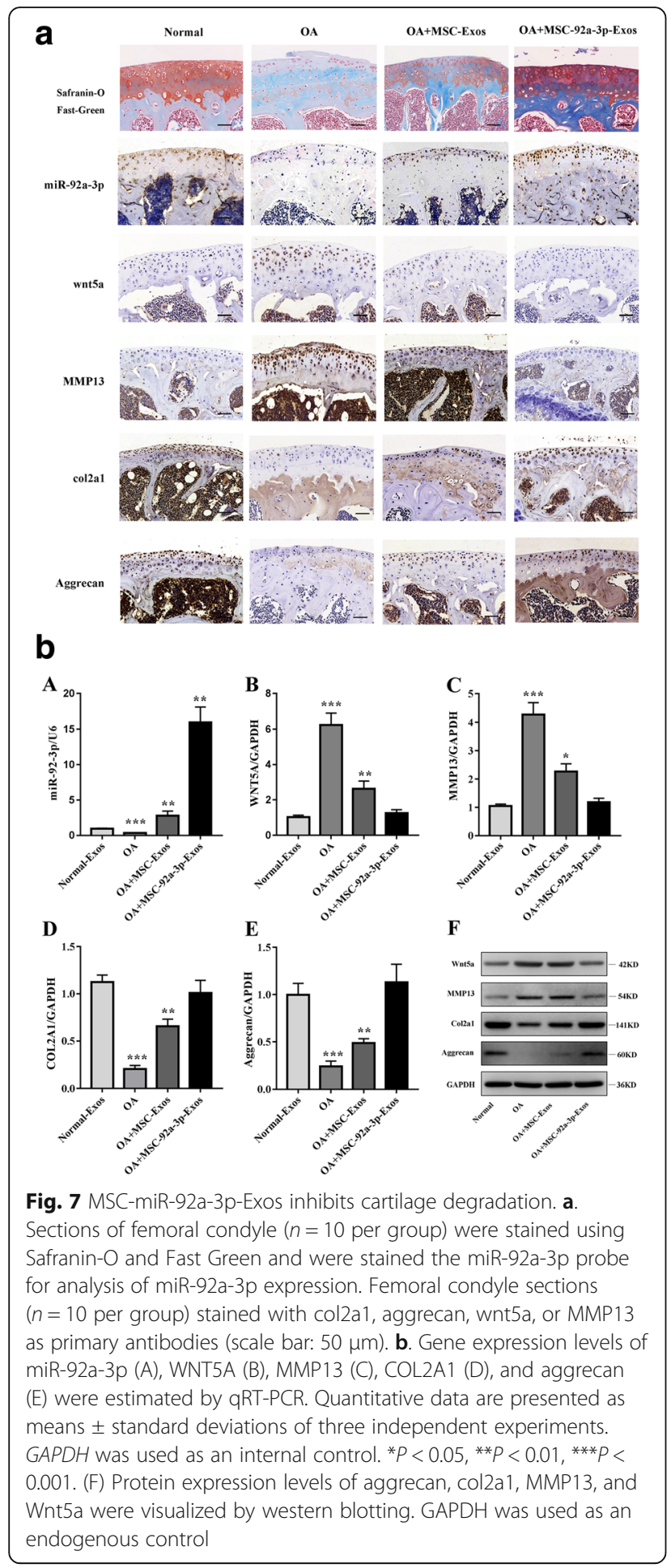

reduced collagen deposition and COL2A1 expression, while treatment with MSC-miR-92a-3p-Exos markedly suppressed WNT5A production and was associated with increased collagen deposition and COL2A1 expression. Therefore, these data suggest that exosomal miR-92a-3p plays an active role in the regulation of WNT5A during chondrogenesis and degeneration.

Wnt proteins affect cellular homeostasis by regulating cell proliferation, cell fate determination and differentiation [43]. WNT5A plays dual roles in matrix homeostasis, including catabolic role and anabolic effect [27, 44, 45]. For example, it has been demonstrated that Wnt5a could induce MMPs expression and inhibit collagen II expression in chondrocytes [27, 44]. However, Kumawat et al. reported that Wnt5a signaling is activated by TGF- $\beta$ and necessary for TGF- $\beta$-induced ECM production in smooth muscle cells [45]. Tao et al. also demonstrated Wnt5a and Wnt5b carried by exosomes activated YAP via the alternative Wnt signaling pathway and enhanced proliferation and migration of chondrocytes with the side-effect of significantly decreasing ECM secretion [15]. In our study, we demonstrated the expression profile of WNT5A during MSC chondrogenesis and found that WNT5A expression was maintained at a stable level in the early stage (days 3-14) of chondrogenic differentiation, which may enhance chondrogenesis. However, expression of WNT5A rapidly increased in the late stage (days 14-21), which may promote MMP13 expression, leading to the loss of aggrecan, COL2A1, COL9A1, and COMP expression. Thus, our data are consistent with previous reports of the dual nature of WNT5A function during cartilage development and in disease. Moreover, PHCs were treated with MSC-miR-92a-3p-Exos or MSC-anti-miR-92a-3p-Exos and found that MSC-miR92a-3p-Exos promote the expression of cartilage matrix proteins, such as aggrecan, COL2A1, and COMP, and suppress the expression of MMP13 and WNT5A. Further, the silencing of WNT5A blocks the catalytic activity of WNT5A and suppresses cartilage degeneration [28]. The Wnt inhibitor M04690 was developed as a potent inhibitor of the Wnt pathway and shows potential as a disease-modifying OA drug (DMOAD) [46]. Interestingly, to some extent, we noted that exosomal miR-92a-3p functions similarly to a Wnt inhibitor. In this study, exosomal miR-92a-3p inhibited WNT5A expression and enhanced aggrecan, COMP, and COL2A1 expression in chondrocytes.

To further explore the role of WNT5A in the regulation of cartilage degradation, we transfected PHCs with siWNT5A and found that COL2A1, COL9A1, aggrecan, and COMP mRNA expression was significantly upregulated, as was SOX9, aggrecan, and COL2A1 protein expression. This suggests that WNT5A may play an important role in cartilage degradation. Luciferase reporter assays showed that miR-92a-3p bound the 3'-UTR of WNT5A mRNA and downregulated its expression at the post-transcriptional level. Overall, our data showed opposing expression patterns between WNT5A and miR-92a-3p, indicating that miR-92a-3p regulates $W N T 5 A$ expression during chondrogenesis. 
Furthermore, in vivo, we investigate the role of MSC-miR-92a-3p-Exos by a collagenase-induced OA mice model. We found and determined that MSC-miR-92a-3p-Exos inhibit the progression of early $\mathrm{OA}$ and prevented the severe damage to knee articular cartilage in the OA mice model.

Although this study demonstrated that exosomal miR-92a-3p is efficacious in cartilage development and degeneration, there are still some limitations to our study. First, the detailed signaling pathways of the dual functions of WNT5A in MSCs undergoing chondrogenesis have not yet been clarified. We plan to conduct a cell signaling study in human bone MSCs to further elaborate the dual regulatory role of WNT5A in chondrogenesis. Furthermore, further studies are needed to clarify the specific function of exosomal-miR-92a-3p in the destabilization of the medial meniscus (DMM) model of OA and osteochondral defect model. For example, studies in rabbits or rats, in which exosome functions could be explored in combination with scaffolds for the treatment of cartilage defects and OA would be useful.

\section{Conclusions}

The present study demonstrated that exosomal miR-92a-3p is expressed before and after chondrogenic differentiation in MSCs and is differentially expressed in OA. We found that exosomal miR-92a-3p functions as a negative regulator by downregulating WNT5A in both chondrogenesis and OA pathogenesis. We suggest that controlling the expression of exosomal miR-92a-3p has potential as a novel approach for prevention and treatment of $\mathrm{OA}$.

\section{Additional file}

Additional file 1: MiRNA expression profiles in exosomes before and after chondrogenic differentiation of MSCs. The expression profiles of miRNAs in exosomes before and after chondrogenic differentiation were detected using a miRNA microarray. SAM statistical software was used to identify differentially expressed miRNAs between undifferentiated MSC-Exos and chondro-differentiated MSC-Exos. Among the miRNAs that were consistently differentially expressed in all three paired samples, 36 were upregulated (fold change $>2$ ) and 105 were downregulated (fold change <-2) in chondro-differentiated MSC-Exos compared to levels in MSC-EXos. (XLSX 69 kb)

\footnotetext{
Abbreviations

3'-UTR: 3'-untranslated region; ADAMTS: A disintegrin and metalloproteinase with thrombospondin motifs; DMEM: Dulbecco's modified Eagle's medium; ECM: Extracellular matrix; hMSC: Human bone marrow-derived MSCs; IL: Interleukin; MSC-Exos: Exosomes secreted by mesenchymal stem cells; miRNA: microRNA; MMP: Matrix metalloproteinase; MSC miR-92a-3pExos: Exosomes secreted by miR-92a-3p-overexpressing mesenchymal stem cells; OA: Osteoarthritis; PHC: Primary human chondrocyte; qRT$P C R$ : quantitative real-time reverse transcription-polymerase chain reaction; TGB: Transforming growth factor
}

\section{Acknowledgments}

We thank Xuerong Li (Department of Parasitology, Zhongshan School of Medicine, Sun Yat-sen University, Guangzhou, China) for his aid with microscopy and other techniques.

\section{Funding}

This study was supported by the National Nature Science Foundation of China (81572119, and 81472101) and the Guangdong Provincial Natural Science Foundation of China (201707010211 and 2015A020212015).

\section{Availability of data and materials}

All data generated or analyzed during this study are included in this published article and Additional file.

\section{Authors' contributions}

YK and WML contributed to the study conception and design, GPM, ZYH, SH, ZJZ, ZQZ, and ZKC performed the experiments. GPM created the graphs and wrote the manuscript. YK revised the manuscript and WML participated in the discussion of the manuscript. All authors analyzed and interpreted experimental data. All authors read and approved the final manuscript.

\section{Ethics approval and consent to participate}

All experimental protocols were approved by the Ethics Committee of the First Affiliated Hospital of Sun Yat-sen University. Written informed consent was obtained from all donors.

\section{Consent for publication}

Not applicable.

\section{Competing interests}

The authors declare that they have no competing interests.

\section{Publisher's Note}

Springer Nature remains neutral with regard to jurisdictional claims in published maps and institutional affiliations.

Received: 28 May 2018 Revised: 30 July 2018

Accepted: 3 September 2018 Published online: 26 September 2018

\section{References}

1. Loeser RF, Goldring SR, Scanzello CR, Goldring MB. Osteoarthritis: a disease of the joint as an organ. Arthritis Rheum. 2012;64:1697-707. https://doi.org/ 10.1002/art.34453.

2. Kobayashi M, Squires GR, Mousa A, Tanzer M, Zukor DJ, Antoniou J, et al. Role of interleukin-1 and tumor necrosis factor alpha in matrix degradation of human osteoarthritic cartilage. Arthritis Rheum. 2005;52:128-35. https:// doi.org/10.1002/art.20776.

3. Daheshia M, Yao JQ. The interleukin 1beta pathway in the pathogenesis of osteoarthritis. J Rheumatol. 2008;35:2306-12.

4. Toh WS, Foldager CB, Pei M, Hui JH. Advances in mesenchymal stem cellbased strategies for cartilage repair and regeneration. Stem Cell Rev. 2014; 10:686-96. https://doi.org/10.1007/s12015-014-9526-z.

5. Meirelles LS, Fontes AM, Covas DT, Caplan Al. Mechanisms involved in the therapeutic properties of mesenchymal stem cells. Cytokine Growth Factor Rev. 2009;20:419-27. https://doi.org/10.1016/j.cytogfr.2009.10.002.

6. Toh WS, Lai RC, Hui J, Lim SK. MSC exosome as a cell-free MSC therapy for cartilage regeneration: implications for osteoarthritis treatment. Semin Cell Dev Biol. 2017;67:56-64. https://doi.org/10.1016/j.semcdb.2016.11.008.

7. Lai RC, Arslan F, Lee MM, Sze NS, Choo A, Chen TS, et al. Exosome secreted by MSC reduces myocardial ischemia/reperfusion injury. Stem Cell Res. 2010;4:214-22. https://doi.org/10.1016/j.scr.2009.12.003.

8. Z Zhang S, Chu WC, Lai RC, Lim SK, Hui JH, Toh WS. Exosomes derived from human embryonic mesenchymal stem cells promote osteochondral regeneration. Osteoarthr Cartil. 2016;24:2135-40. https://doi.org/10.1016/j. joca.2016.06.022.

9. Vonk LA, van Dooremalen S, Liv N, Klumperman J, Coffer PJ, Saris D, et al. Mesenchymal stromal/stem cell-derived extracellular vesicles promote human cartilage regeneration in vitro. Theranostics. 2018;8:906-20. https:// doi.org/10.7150/thno.20746.

10. Zhang S, Chuah SJ, Lai RC, Hui J, Lim SK, Toh WS. MSC exosomes mediate cartilage repair by enhancing proliferation, attenuating apoptosis and 
modulating immune reactivity. Biomaterials. 2018;156:16-27. https://doi.org/ 10.1016/j.biomaterials.2017.11.028

11. Xin H, Li Y, Chopp M. Exosomes/miRNAs as mediating cell-based therapy of stroke. Front Cell Neurosci. 2014;8:377. https://doi.org/10.3389/fncel.2014. 00377.

12. Burger D, Vinas JL, Akbari S, Dehak H, Knoll W, Gutsol A, et al. Human endothelial colony-forming cells protect against acute kidney injury: role of exosomes. Am J Pathol. 2015;185:2309-23. https://doi.org/10.1016/j.ajpath. 2015.04.010.

13. Carthew RW, Sontheimer EJ. Origins and mechanisms of miRNAs and siRNAs. Cell. 2009;136:642-55. https://doi.org/10.1016/j.cell.2009.01.035

14. Mayourian J, Ceholski DK, Gorski PA, Mathiyalagan P, Murphy JF, Salazar SI, et al. Exosomal microRNA-21-5p Mediates Mesenchymal Stem Cell Paracrine Effects on Human Cardiac Tissue Contractility. Circ Res. 2018;122:933-44. https://doi.org/10.1161/CIRCRESAHA.118.312420.

15. Tao SC, Yuan T, Zhang YL, Yin WJ, Guo SC, Zhang CQ. Exosomes derived from miR-140-5p-overexpressing human synovial mesenchymal stem cells enhance cartilage tissue regeneration and prevent osteoarthritis of the knee in a rat model. Theranostics. 2017;7:180-95. https://doi.org/10.7150/thno. 17133.

16. Staines KA, Macrae VE, Farquharson C. Cartilage development and degeneration: a Wnt Wnt situation. Cell Biochem Funct. 2012;30:633-42. https://doi.org/10.1002/cbf.2852.

17. Ge X, Shi R, Ma X. The secreted protein WNT5A regulates condylar chondrocyte proliferation, hypertrophy and migration. Arch Oral Biol. 2017; 82:171-9. https://doi.org/10.1016/j.archoralbio.2017.06.019.

18. Lerner UH, Ohlsson C. The WNT system: background and its role in bone. J Intern Med. 2015;277:630-49. https://doi.org/10.1111/joim.12368.

19. Chun JS, Oh H, Yang S, Park M. Wnt signaling in cartilage development and degeneration. BMB Rep. 2008;41:485-94.

20. Maeda K, Kobayashi Y, Udagawa N, Uehara S, Ishihara A, Mizoguchi T, et al. Wnt5a-Ror2 signaling between osteoblast-lineage cells and osteoclast precursors enhances osteoclastogenesis. Nat Med. 2012;18:405-12. https:// doi.org/10.1038/nm.2653.

21. Hosseini-Farahabadi S, Geetha-Loganathan P, Fu K, Nimmagadda S, Yang HJ, Richman JM. Dual functions for WNT5A during cartilage development and in disease. Matrix Biol. 2013;32:252-64. https://doi.org/10.1016/j.matbio.2013. 02.005 .

22. Kawakami Y, Wada N, Nishimatsu SI, Ishikawa T, Noji S, Nohno T. Involvement of Wnt-5a in chondrogenic pattern formation in the chick limb bud. Develop Growth Differ. 1999;41:29-40.

23. Church V, Nohno T, Linker C, Marcelle C, Francis-West P. Wnt regulation of chondrocyte differentiation. J Cell Sci. 2002;115:4809-18.

24. Yang $Y$, Topol L, Lee $H$, Wu J. Wnt5a and Wnt5b exhibit distinct activities in coordinating chondrocyte proliferation and differentiation. Development. 2003;130:1003-15.

25. Huang G, Chubinskaya S, Liao W, Loeser RF. Wnt5a induces catabolic signaling and matrix metalloproteinase production in human articular chondrocytes. Osteoarthr Cartil. 2017;25:1505-15. https://doi.org/10.1016/j. joca.2017.05.018

26. Ge XP, Gan YH, Zhang CG, Zhou CY, Ma KT, Meng JH, et al. Requirement of the NF-kappaB pathway for induction of Wnt-5A by interleukin-1beta in condylar chondrocytes of the temporomandibular joint: functional crosstalk between the Wnt-5A and NF-kappaB signaling pathways. Osteoarthr Cartil. 2011;19:111-7. https://doi.org/10.1016/j.joca.2010.10.016.

27. Ge X, Ma X, Meng J, Zhang C, Ma K, Zhou C. Role of Wnt-5A in interleukin1 beta-induced matrix metalloproteinase expression in rabbit temporomandibular joint condylar chondrocytes. Arthritis Rheum. 2009;60: 2714-22. https://doi.org/10.1002/art.24779.

28. Shi S, Man Z, Li W, Sun S, Zhang W. Silencing of Wnt5a prevents interleukin1 beta-induced collagen type II degradation in rat chondrocytes. Exp Ther Med. 2016;12:3161-6. https://doi.org/10.3892/etm.2016.3788.

29. Ning G, Liu X, Dai M, Meng A, Wang Q. MicroRNA-92a upholds bmp signaling by targeting noggin3 during pharyngeal cartilage formation. Dev Cell. 2013;24:283-95. https://doi.org/10.1016/j.devcel.2012.12.016.

30. Mao G, Wu P, Zhang Z, Zhang Z, Liao W, Li Y, et al. MicroRNA-92a-3p regulates Aggrecanase-1 and Aggrecanase-2 expression in Chondrogenesis and IL-1beta-induced catabolism in human articular chondrocytes. Cell Physiol Biochem. 2017;44:38-52. https://doi.org/10.1159/000484579.

31. Mao G, Zhang Z, Huang Z, Chen W, Huang G, Meng F, et al. MicroRNA-92a$3 p$ regulates the expression of cartilage-specific genes by directly targeting histone deacetylase 2 in chondrogenesis and degradation. Osteoarthr Cartil. 2017;25:521-32. https://doi.org/10.1016/j.joca.2016.11.006.

32. Meng FG, Zhang ZQ, Huang GX, Chen WS, Zhang ZJ, He AS, et al. Chondrogenesis of mesenchymal stem cells in a novel hyaluronate-collagentricalcium phosphate scaffolds for knee repair. Eur Cell Mater. 2016;31:79-94.

33. Greening DW, Xu R, Ji H, Tauro BJ, Simpson RJ. A protocol for exosome isolation and characterization: evaluation of ultracentrifugation, densitygradient separation, and immunoaffinity capture methods. Methods Mol Biol. 2015;1295:179-209. https://doi.org/10.1007/978-1-4939-2550-6_15.

34. Zhu Y, Wang Y, Zhao B, Niu X, Hu B, Li Q, et al. Comparison of exosomes secreted by induced pluripotent stem cell-derived mesenchymal stem cells and synovial membrane-derived mesenchymal stem cells for the treatment of osteoarthritis. Stem Cell Res Ther. 2017;8:64. https://doi.org/10.1186/ s13287-017-0510-9.

35. Chen D, Shen J, Zhao W, Wang T, Han L, Hamilton JL, et al. Osteoarthritis: toward a comprehensive understanding of pathological mechanism. Bone Res. 2017:5:16044. https://doi.org/10.1038/boneres.2016.44.

36. Mathiessen A, Conaghan PG. Synovitis in osteoarthritis: current understanding with therapeutic implications. Arthritis Res Ther. 2017;19:18. https://doi.org/10.1186/s13075-017-1229-9.

37. Yu SP, Hunter DJ. Intra-articular therapies for osteoarthritis. Expert Opin Pharmacother. 2016;17:2057-71. https://doi.org/10.1080/14656566.2016. 1232396.

38. Glyn-Jones S, Palmer AJ, Agricola R, Price AJ, Vincent TL, Weinans H, et al. Osteoarthritis. Lancet. 2015;386:376-87. https://doi.org/10.1016/S01406736(14)60802-3.

39. Chen TS, Lim SK. Measurement of precursor miRNA in exosomes from human ESC-derived mesenchymal stem cells. Methods Mol Biol. 2013;1024: 69-86. https://doi.org/10.1007/978-1-62703-453-1_6.

40. Chen TS, Lai RC, Lee MM, Choo AB, Lee CN, Lim SK. Mesenchymal stem cell secretes microparticles enriched in pre-microRNAs. Nucleic Acids Res. 2010; 38:215-24. https://doi.org/10.1093/nar/gkp857.

41. Meng F, Zhang Z, Chen W, Huang G, He A, Hou C, et al. MicroRNA-320 regulates matrix metalloproteinase-13 expression in chondrogenesis and interleukin-1 beta-induced chondrocyte responses. Osteoarthr Cartil. 2016;24: 932-41. https://doi.org/10.1016/j.joca.2015.12.012.

42. Ham O, Song BW, Lee SY, Choi E, Cha MJ, Lee CY, et al. The role of microRNA-23b in the differentiation of MSC into chondrocyte by targeting protein kinase a signaling. Biomaterials. 2012;33:4500-7. https://doi.org/10. 1016/j.biomaterials.2012.03.025

43. Logan CY, Nusse R. The Wnt signaling pathway in development and disease. Annu Rev Cell Dev Biol. 2004;20:781-810. https://doi.org/10.1146/ annurev.cellbio.20.010403.113126.

44. Ryu JH, Chun JS. Opposing roles of WNT-5A and WNT-11 in interleukin1 beta regulation of type II collagen expression in articular chondrocytes. J Biol Chem. 2006;281:22039-47. https://doi.org/10.1074/jbc.M601804200.

45. Kumawat K, Menzen MH, Bos IS, Baarsma HA, Borger P, Roth M, et al. Noncanonical WNT-5A signaling regulates TGF-beta-induced extracellular matrix production by airway smooth muscle cells. FASEB J. 2013;27:1631-43. https://doi.org/10.1096/fj.12-217539.

46. Yazici Y, McAlindon TE, Fleischmann R, Gibofsky A, Lane NE, Kivitz AJ, et al. A novel Wnt pathway inhibitor, SM04690, for the treatment of moderate to severe osteoarthritis of the knee: results of a 24-week, randomized, controlled, phase 1 study. Osteoarthr Cartil. 2017;25:1598-606. https://doi. org/10.1016/j.joca.2017.07.006.

\section{Ready to submit your research? Choose BMC and benefit from:}

- fast, convenient online submission

- thorough peer review by experienced researchers in your field

- rapid publication on acceptance

- support for research data, including large and complex data types

- gold Open Access which fosters wider collaboration and increased citations

- maximum visibility for your research: over $100 \mathrm{M}$ website views per year

At BMC, research is always in progress.

Learn more biomedcentral.com/submissions 